\title{
Metallographic Preparation Techniques for Evaluation of Co-Cr-Mo Alloys
}

\author{
C. McNee, J.J. Frafjord, and M. Mondo
}

IMR KHA-Portland, 5687-A SE International Way Portland, OR 97222

Metallographic preparation and subsequent microstructural examination of cobalt-chromiummolybdenum (Co-Cr-Mo) alloys has proven challenging due to the high hardness of the material and the tendency toward surface aberrations during polishing procedures. Due to the rapid passivation of these alloys after final polishing, etching can also pose a unique set of problems. This study explores various grinding, polishing and etching procedures for both cast and forged samples. Comparison of these methods will be demonstrated by the integrity of microstructural features via light microscopy and electron microscopy and by the ease of various image analysis techniques.

This alloy system is used extensively in the medical implant industry due to biocompatibility. CoCr-Mo stands out among the other biomedical metals as being the most wear resistant and finds many applications in high wear scenarios, i.e. femoral head in prosthetic hips [1]. Due to the prevalent use of Co-Cr-Mo throughout the biomedical field, proper identification and interpretation of microstructural features is paramount.

The Co-Cr-Mo alloys present a unique set of polishing conditions and while diamond polish requires long polishing steps, alumina can cause surface aberrations. These alloys also passivate after the final polishing step, and this passivation or 'burning' reveals scratches and general surface damage when the samples are subsequently etched. This study will demonstrate the relationships between time and surface roughness for these different polishing techniques.

Different etching techniques will be studied to compare the evaluation of grain boundaries, carbides, porosity, dendritic features in cast, burning around edges, and effect on image analysis routines. Etching techniques previously described in handbooks and journals will be evaluated. In one example, the carbide network within the dendritic structure is easily demonstrated using a ferric chloride electrolytic etch (see Figure 1). However, if image analysis is required for quantitative evaluation of the carbides, a $2 \%$ nital electrolytic etch is preferred (see Figure 2). The nital electrolytic etch creates a more planar surface that is more conducive to image analysis gray thresholding routines.

\section{Reference:}

[1] M. Niinomi, "Recent Metallic Materials for Biomedical Applications," Metallurgical and Materials Transactions A, Vol. 33A (March 2002). 


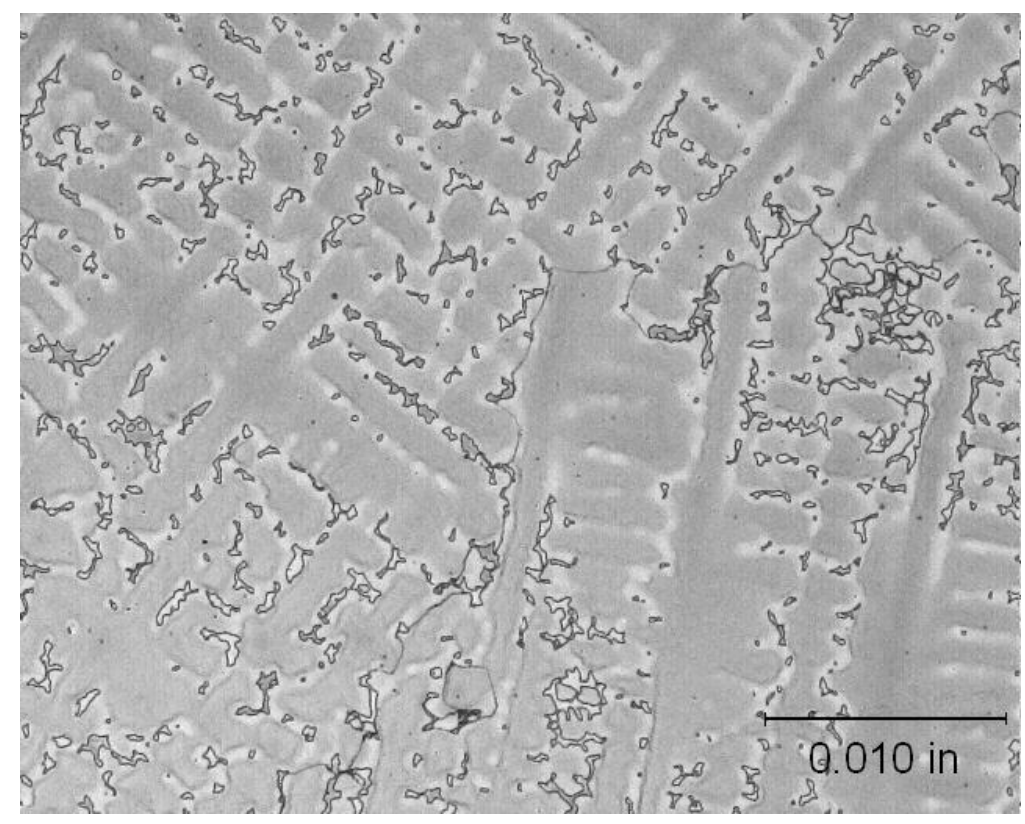

Figure 1: As-cast cobalt alloy sample electrolytically etched in a ferric chloride solution showing carbides and dendritic structure.

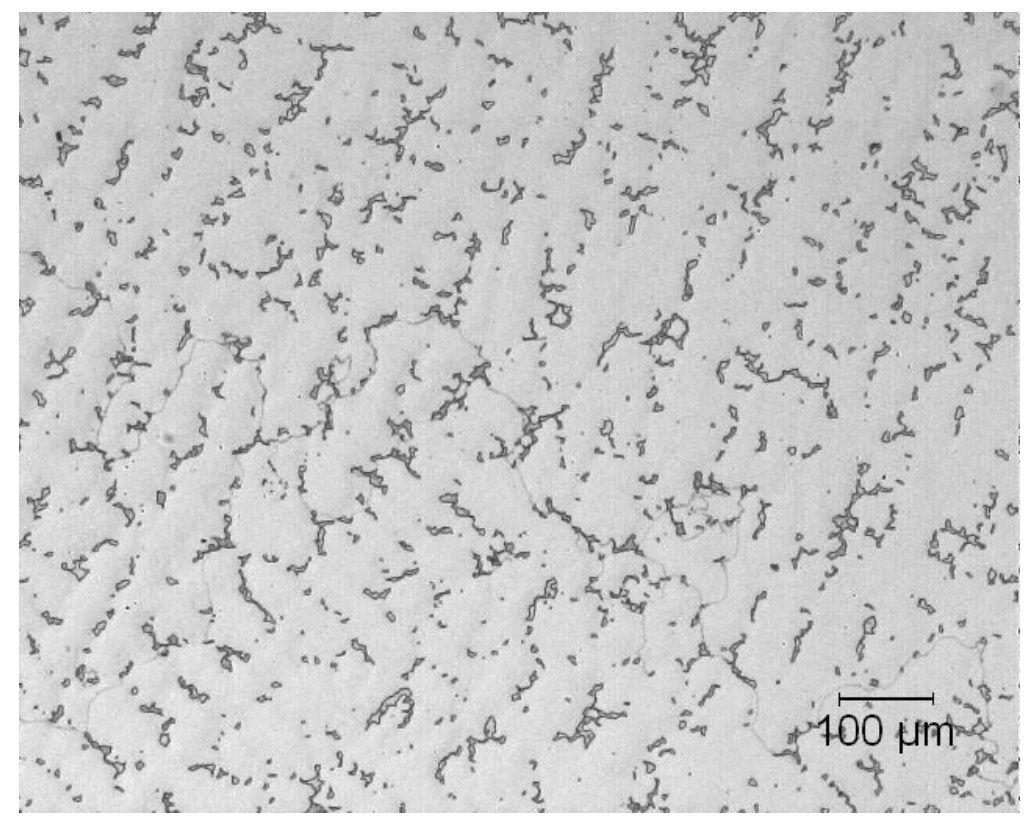

Figure 2: Same as-cast cobalt alloy material electrolytically etched in a nital solution showing carbides without the dendritic structure, which is better suited to image analysis. 